\title{
Modelling the pan-spectral energy distributions of Starburst and active galaxies
}

\author{
M. A. Dopita ${ }^{1}$, J. Fischera ${ }^{1}$, B. Groves ${ }^{1}$, R. S. Sutherland ${ }^{1}$, \\ L. J. Kewley ${ }^{2}$, R. Tuffs ${ }^{3}$, C. Popescu ${ }^{3}$ and C. Leitherer ${ }^{4}$ \\ ${ }^{1}$ Research School of Astronomy \& Astrophysics, Australia National University, Canberra, \\ Australia email: Michael.Dopita@anu.edu.au \\ ${ }^{2}$ Harvard-Smithsonian Center for Astrophysics, Cambridge, MA, USA \\ ${ }^{3}$ MPI für Kernphysik, Heidelberg, Germany \\ ${ }^{4}$ Space Telescope Science Institure, Baltimore, MD, USA
}

\begin{abstract}
We present results of a self-consistent model of the spectral energy distribution (SED) of starburst galaxies. Two parameters control the IR SED, the mean pressure in the ISM and the destruction timescale of molecular clouds. Adding a simplified AGN spectrum provides mixing lines on IRAS color : color diagrams. This reproduces the observed colors of both AGNs and starbursts.
\end{abstract}

\section{SED Modelling}

Our model, which will be described in detail elsewhere (Dopita et al. 2004), combines the output of the stellar spectral synthesis code STARBURST 99 as input to the nebular modelling code MAPPINGS IIIq, including a 1-D dynamical evolution model of HII regions around massive clusters of young stars. This model includes all relevant dust and gas physics to provide the nebular line, continuum and dust re-emission spectrum. This allows us to produce purely theoretical synthetic spectral energy distributions (SEDs) in the $0.09-1000 \mu \mathrm{m}$ wavelength range.

We have applied this code to make models of solar metallicity starbursts lasting some $10^{8}$ years. We find that the expansion of the Hir region "stalls" when the internal pressure matches the pressure of the ambient ISM, c.f. Oey \& Clarke (1998). Dust in high pressure environments is closer to the exciting stars, and hotter, so that the far-IR SED peaks at shorter wavelengths. In addition, the covering factor of the molecular clouds with respect to the central stars largely determines the strength of the PAH emission features in the $3-30 \mu \mathrm{m}$ band, since the PAHs are destroyed in the diffuse and ionized phases. Molecular clouds around HII regions are destroyed and dispersed over time. As a result, the starburst SED shape is largely controlled by this molecular cloud dissipation timescale which determines the visibility of the central cluster at UV wavelengths and the strength of the PAH emission bands in the IR. By contrast, the ambient ISM pressure determines the SED mainly by controlling the dust temperature, which affects the peak of the FIR dust re-emission feature.

Our models use a solar abundance set (as modified by the latest abundance determinations of Allende Prieto et al. (2002), and references therein) with depletion factors given by Dopita et al. (2000). To reproduce the PAH emission features, we require that $\sim 70 \%$ of the $\mathrm{C}$ is locked up in PAHs. As a consequence, when PAHs are destroyed by photodisscociation, the $2200 \AA$ absorption feature becomes very weak, and the resulting theoretical attenuation curve looks almost identical to the Calzetti (2001) curve determined empirically for starbursts. 

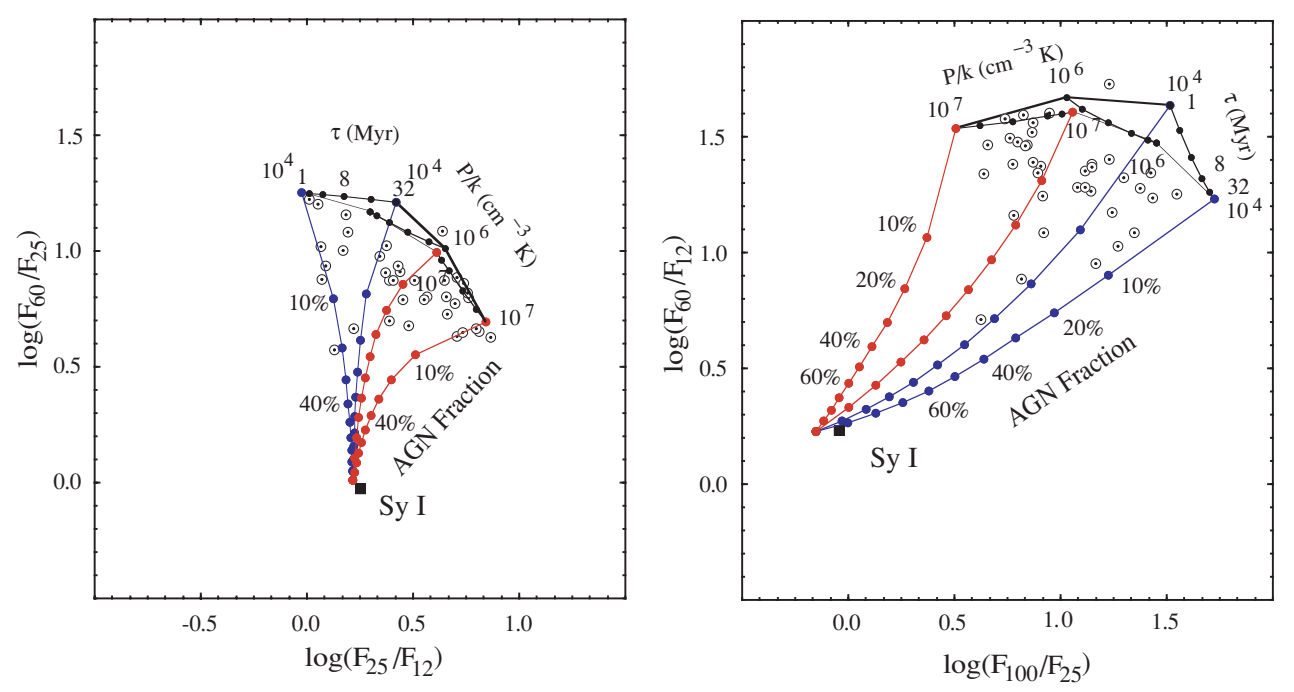

Figure 1. IRAS color : color diagrams for the Rush et al. (1993) starbursts compared with our models. We also show mixing lines with our AGN model. The observed starbursts may contain up to $10 \%$ of an AGN contribution.

Here, we have also added an AGN contribution, assumed to be Synchrotron selfCompton emission from a trapped jet, becoming Synchrotron self-absorbed in the $10-$ $100 \mu \mathrm{m}$ wavelength band. For a "pure" AGN, this model reproduces the Dopita et al. (1998) mean Seyfert I colors, derived from the Rush et al. (1993) IRAS dataset. Figure 1 shows IRAS color : color diagrams for various AGN contributions, expressed as a percentage of the bolometric flux of the starburst.

We can conclude that our models reproduce the range of FIR colors seen in starburst galaxies, and that objects classified as starbursts may in fact contain up to $10 \%$ contribution from an AGN.

\section{Acknowledgements}

Dopita acknowledges support through a Federation Fellowship. Dopita, Sutherland \& Fishera acknowlege ARC Discovery project DP0208445. Kewley is supported by a Harvard-Smithsonian CfA Fellowship.

\section{References}

Allende Prieto, C., Lambert, D. L., \& Asplund, M. 2001, ApJL, in press Calzetti, D. 2001, PASP, 113, 1449

Dopita M. A., Heisler, C. A., Lumsden, S., \& Bailey, J. 1998, ApJ, 498, 570

Dopita, M. A., Kewley, L. J., Heisler, C. A., \& Sutherland, R. S. 2000, ApJ, 542, 224

Dopita, M. A., et al. 2004, ApJ, in press

Oey, M. S., \& Clarke, C. J. 1998, AJ, 115, 1543

Rush, B., Malkan, M. A., \& Spinoglio, L. 1993, ApJS, 89, 1 